\title{
EXISTENCE OF MAXIMAL ELEMENT AND EQUILIBRIUM FOR A NONPARACOMPACT N-PERSON GAME
}

\author{
WON KYU KIM
}

(Communicated by George C. Papanicolaou)

\begin{abstract}
In this paper, we will introduce the concept of $L_{s}$-majorized correspondence and prove a new maximal element existence theorem on nonparacompact sets. As applications, we prove a new existence theorem of equilibrium for a nonparacompact 1-person game with $L_{s}$-majorized preference correspondences, and then we prove that a nonparacompact $\mathrm{N}$-person game with preference correspondences of class $L$ can be reduced to a 1-person game with $L_{s}$-majorized preference correspondences.
\end{abstract}

\section{INTRODUCTION}

In the last twenty years, the classical Arrow-Debreu [2] result on the existence of Walrasian equilibria has been generalized in many directions. Bewley [3] proved an equilibrium existence theorem for economies with an infinite dimensional commodity space and Mas-Colell [17] was first to show that the existence of equilibrium can be established without assuming preferences to be total or transitive. Next, by using a maximal element existence theorem, Gale and Mas-Colell [13] gave a proof of the existence of a competitive equilibrium without ordered preferences. Shafer and Sonnenschein [19] gave results in the same direction and they proved the Arrow-Debreu lemma for abstract economies' for the case where preferences may not be total or transitive. On the other hand, Borglin and Keiding [4] proved a new existence theorem for a compact abstract economy with KF-majorized preference correspondences, and following their idea, till now there have been a number of generalizations of the existence of equilibria for compact abstract economies.

In 1983, Yannelis and Prabhakar [26] proved the existence of maximal elements over compact subsets of Hausdorff topological vector spaces generalizing the previous results of Fan [8], Sonnenschein [21], Borglin-Keiding [4], and Aliprantis-Brown [1], and they also proved the existence of an equilibrium for an abstract economy defined in Shafer-Sonnenschein [19] and Borglin-Keiding

Received by the editors August 30, 1989 and, in revised form, April 5, 1991; presented at the 2nd International Conference on Fixed Point Theory and Applications in Halifax, June 9-14, 1991.

1991 Mathematics Subject Classification. Primary 90A14; Secondary 52A07, 90D06.

Key words and phrases. Maximal element, equilibrium, N-person game, $L_{s}$-majorized correspondence, class $L$, upper semicontinuous.

This work was partially supported by a KOSEF grant in 1991. 
[4]. Recently, Ding-Kim-Tan [5, 6] proved some existence theorems of equilibria for noncompact abstract economies with $L^{*}$-majorized preferences.

As in $[4,13,17,19,23,26]$, in most results on the existence of equilibria for abstract economies, the underlying spaces (commodity spaces or choice sets) are always compact and convex. However, in recent papers $[5,6,18,24,25]$, the underlying spaces are not always compact, but the paracompactness is needed. Moreover, it should be noted that we will encounter many kinds of preferences in various economic situations; so it is important that we consider several types of preferences and obtain some existence results for such correspondences in noncompact (or nonparacompact) settings.

The purpose of this paper is twofold. First, we will introduce the concept of $L_{s}$-majorized correspondence and prove a new maximal element existence theorem on nonparacompact sets. Second, as applications we prove a new existence theorem of equilibrium for a nonparacompact 1-person game with $L_{s}$ majorized preference correspondences, and then we prove that a nonparacompact $\mathrm{N}$-person game with preference correspondences of class $L$ can be reduced to a 1 -person game with $L_{s}$-majorized preference correspondences.

\section{Preliminaries}

Let $A$ be a subset of a topological space $X$. We shall denote by $2^{A}$ the family of all subsets of $A$ and by $\operatorname{cl} A$ the closure of $A$ in $X$. If $A$ is a subset of a vector space, we shall denote by $\operatorname{con} A$ the convex hull of $A$. If $A$ is a nonempty subset of a topological vector space $X$ and $S, T: A \rightarrow 2^{X}$ are correspondences, then con $T, \mathrm{cl} T, T \cap S: A \rightarrow 2^{X}$ are correspondences defined by $(\operatorname{con} T)(x)=\operatorname{con} T(x),(\operatorname{cl} T)(x)=\operatorname{cl} T(x)$, and $(T \cap S)(x)=T(x) \cap S(x)$ for each $x \in A$, respectively.

In [26] Yannelis and Prabhakar introduced the following definition, which generalizes KF correspondences defined in [4, p. 313] and is useful for many applications in mathematical economics (i.e., see [4-6, 23-26]):

Let $X$ be a nonempty subset of a topological vector space. A correspondence $\phi: X \rightarrow 2^{X}$ is said to be of class $L$ [26] if (i) for each $x \in X, x \notin \operatorname{con} \phi(x)$, (ii) for each $y \in X, \phi^{-1}(y)=\{x \in X: y \in \phi(x)\}$ is open in $X$.

We now introduce the following definition, which generalizes the correspondence of class $L$.

Definition. Let $X$ be a nonempty subset of a topological vector space and $x \in$ $X$. Let $\phi: X \rightarrow 2^{X}$ be a given correspondence. A correspondence $\phi_{x}: X \rightarrow 2^{X}$ is said to be an $L_{s}$-majorant of $\phi$ at $x$ if there exists an open neighborhood $N_{x}$ of $x$ in $X$ such that (a) for each $z \in X, \phi(z) \subset \phi_{x}(z)$, (b) for each $z \in N_{x}, z \notin \operatorname{con} \phi_{x}(z)$, and (c) for each $y \in X, \phi_{x}^{-1}(y)$ is open in $X$. The correspondence $\phi$ is said to be $L_{s}$-majorized if for each $x \in X$ with $\phi(x) \neq \varnothing$, there exists an $L_{s}$-majorant of $\phi$ at $x$.

It is easy to see that every KF correspondence is a correspondence of class $L$ and every correspondence of class $L$ is clearly $L_{s}$-majorized. Note that an $L_{s}$-majorized correspondence is clearly an $L$-majorized correspondence defined in [26, p. 240], i.e., our definition has one more extra condition (a). However as in many applications $[5,6,18,23,24]$ the maximal element existence theorems for $L$-majorized correspondences always need the compactness (or paracompactness) of the given set $X$, but we shall not need any paracompact- 
ness condition of the set $X$. And it should be noted that if $\phi$ is $L_{s}$-majorized in $X$, then $x \notin \operatorname{con} \phi(x)$ for all $x \in X$.

The following simple example shows an $L_{s}$-majorized correspondence that is not of class $L$.

Example. Let $X=[0,1)$ and $\phi: X \rightarrow 2^{X}$ be defined by

$$
\phi(x)= \begin{cases}\left\{y \in X: 0 \leq y \leq x^{2}\right\}, & x \in(0,1), \\ \varnothing, & x=0 .\end{cases}
$$

Then $\phi$ is not of class $L$ since $\phi^{-1}(y)$ is not open in $X$ for any $y \in(0,1)$. For any $x \in(0,1)$, let $N_{x}=X$ be an open neighborhood of $x$ in $X$, and define $\phi_{x}: X \rightarrow 2^{X}$ by

$$
\phi_{x}(z)=\left\{\begin{array}{l}
\{y \in X: 0 \leq y<x\}, \quad z \in(0,1), \\
\varnothing, \quad z=0 .
\end{array}\right.
$$

Then it is easy to see that $\phi_{x}$ is an $L_{s}$-majorant of $\phi$ at $x$, and hence $\phi$ is a $L_{s}$-majorized correspondence.

Now we recall the following general definitions of equilibrium theory in mathematical economics. Let $I$ be a finite or an infinite set of agents. For each $i \in I$, let $X_{i}$ be a nonempty set of actions. A generalized game (or an abstract economy) $\Gamma=\left(X_{i}, A_{i}, B_{i}, P_{i}\right)_{i \in I}$ is defined as a family of ordered quadruple $\left(X_{i}, A_{i}, B_{i}, P_{i}\right)$ where $X_{i}$ is a nonempty topological vector space (a choice set), $A_{i}, B_{i}: \prod_{j \in I} X_{j} \rightarrow 2^{X_{i}}$ are constraint correspondences and $P_{i}: \prod_{j \in I} X_{j} \rightarrow 2^{X_{i}}$ is a preference correspondence. An equilibrium for $\Gamma$ is a point $\hat{x} \in X=\prod_{i \in I} X_{i}$ such that for each $i \in I, \hat{x}_{i} \in \operatorname{cl} B_{i}(\hat{x})$ and $P_{i}(\hat{x}) \cap A_{i}(\hat{x})=\varnothing$. When $A_{i}=B_{i}$ for each $i \in I$, our definitions of an abstract economy and an equilibrium coincide with the standard definitions, e.g., in $[4,19,23,24,26]$. For each $i \in I, P_{i}^{\prime}: X \rightarrow 2^{X}$ will denote the correspondence defined by $P_{i}^{\prime}(x)=\left\{y \in X: y_{i} \in P_{i}(x)\right\}\left(=\pi_{i}^{-1}\left(P_{i}(x)\right)\right.$, where $\pi_{i}: X \rightarrow X_{i}$ is the $i$ th projection). In particular, when $I=\{1, \ldots, n\}$, we may call $\Gamma$ an $N$-person game.

\section{EXISTENCE OF A MAXIMAL ELEMENT}

We shall first need the following lemma, which is a generalization of earlier Fan's maximal element existence theorem appeared in [4, p. 313] and also represents a maximal element version of his recent intersection result [9, Theorem 4]. For completeness, we give a proof.

Lemma. Let $X$ be a nonempty convex subset of a Hausdorff topological vector space and $T: X \rightarrow 2^{X}$ be a correspondence such that for each $x \in X, x \notin$ con $T(x)$ and for each $y \in X, T^{-1}(y)$ is open in $X$. Suppose further that there exists a nonempty compact subset $D$ of $X$ and a nonempty compact convex subset $X_{0}$ of $X$ such that $X_{0} \cap \operatorname{con} T(x) \neq \varnothing$ for all $x \in X \backslash D$.

Then there exists a point $\hat{x} \in X$ such that $T(\hat{x})=\varnothing$.

Proof. Suppose that the conclusion is false; then for each $x \in X$ we would have $T(x) \neq \varnothing$ so that $\operatorname{con} T(x) \neq \varnothing$. By Lemma 5.1 in [26], $(\operatorname{con} T)^{-1}(y)$ is open in $X$ for each $y \in X$. Now we define a correspondence

$$
F(x):=X \backslash(\operatorname{con} T)^{-1}(x) \text { for each } x \in X \text {. }
$$


Then for each $x \in X, F(x)$ is closed in $X$. Now we claim that $\bigcap_{x \in X_{0}} F(x) \subset$ $D$. Suppose the contrary, i.e., there exists a point $y \in \bigcap_{x \in X_{0}} F(x)$ such that $y \in X \backslash D$. Then we have

$$
\begin{aligned}
y \in \bigcap_{x \in X_{0}} F(x) & \Leftrightarrow y \in \bigcap_{x \in X_{0}}\left(X \backslash(\cos T)^{-1}(x)\right) \\
& \Leftrightarrow y \in X \backslash \bigcup_{x \in X_{0}}(\operatorname{con} T)^{-1}(x) \\
& \Leftrightarrow \quad X_{0} \cap(\operatorname{con} T)(y)=\varnothing,
\end{aligned}
$$

which contradicts the assumption. Therefore $\bigcap_{x \in X_{0}} F(x)$ is a closed subset of a compact set $D$ so that $\bigcap_{x \in X_{0}} F(x)$ is compact. Since con $T(x) \neq \varnothing$ for each $x \in X$, we have $\bigcup_{x \in X}(\operatorname{con} T)^{-1}(x)=X$, so that

$$
\bigcap_{x \in X} F(x)=\bigcap_{x \in X}\left(X \backslash(\operatorname{con} T)^{-1}(x)\right)=X \backslash \bigcup_{x \in X}(\operatorname{con} T)^{-1}(x)=\varnothing .
$$

Therefore, by Theorem 4 in [9], $F$ cannot be a KKM-map, so that there exists a convex combination $\omega=\sum_{i=1}^{n} \lambda_{i} x_{i} \notin \bigcup_{i=1}^{n} F\left(x_{i}\right)$. Hence we have $\omega \in$ $X \backslash \bigcup_{1=1}^{n} F\left(x_{i}\right)=\bigcap_{i=1}^{n}(\operatorname{con} T)^{-1}\left(x_{i}\right)$, so that $\omega \in(\operatorname{con} T)^{-1}\left(x_{i}\right)$ for each $i=$ $1, \ldots, n$. Therefore $x_{i} \in \operatorname{con} T(\omega)$ for each $i=1, \ldots, n$. Since $\operatorname{con} T(\omega)$ is convex, we have $\omega=\sum_{i=1}^{n} \lambda_{i} x_{i} \in \operatorname{con} T(\omega)$, which contradicts the assumption. Q.E.D.

The existence of maximal element is essential in proving existence of equilibria in generalized games as in [4-6, 18, 23-26]. Recently, using Fan's intersection theorem [9] or the fixed point theorem as in [26], several authors further generalize maximal element existence results in the paracompact settings (i.e., see $[5,6,18,23,24])$.

By modifying the method in [5], we shall prove the following new maximal element existence theorem for $L_{s}$-majorized correspondences without assuming the paracompactness of the given set $X$, which is essential for the existence of equilibria for a nonparacompact 1-person game.

Theorem 1. Let $X$ be a nonempty convex subset of a Hausdorff topological vector space, $D$ be a nonempty compact subset of $X$, and $y_{0} \in D$. Let $\phi: X \rightarrow 2^{X}$ be an $L_{s}$-majorized correspondence such that for each $x \in X \backslash D, y_{0} \in \operatorname{con} \phi(x)$.

Then there exists a maximal element $\hat{x} \in X$, i.e., $\phi(\hat{x})=\varnothing$.

Proof. Suppose that for each $x \in X, \phi(x) \neq \varnothing$. Since $\phi$ is $L_{s}$-majorized, for each $x \in D$, there exists an $L_{s}$-majorant $\phi_{x}$ and an open neighborhood $N_{x}$ of $x$ in $X$ such that

(i) for each $z \in X, \phi(z) \subset \phi_{x}(z)$,

(ii) for each $z \in N_{x}, z \notin \operatorname{con} \phi_{x}(z)$,

(iii) for each $y \in X, \phi_{x}^{-1}(y)$ is open in $X$.

The family $\left\{N_{x}: x \in D\right\}$ is an open covering of $D$, which by the compactness of $D$, contains a finite subcover $\left\{N_{x_{i}}: i \in I\right\}$, where $I$ is a finite set. Then $U=\bigcup_{i \in I} N_{x_{i}}$ is an open set in $X$ containing $D$. For each $i \in I$, denote by $\operatorname{con} \phi_{x_{i}}^{\prime}$ the restriction of $\operatorname{con} \phi_{x_{i}}$ on $U$. Then for each $i \in I$ and for each $y \in X$,

$$
\begin{aligned}
\left(\operatorname{con} \phi_{x_{i}}^{\prime}\right)^{-1}(y) & =\left\{z \in U: y \in \operatorname{con} \phi_{x_{1}}^{\prime}(z)\right\} \\
& =\left\{z \in U: y \in \operatorname{con} \phi_{x_{i}}(z)\right\}=U \cap\left(\operatorname{con} \phi_{x_{i}}\right)^{-1}(y)
\end{aligned}
$$


is open in $X$ by Lemma 5.1 in [26] and condition (iii). Now we define $\Phi: X \rightarrow 2^{X}$ by

$$
\Phi(z)=\left\{\begin{array}{l}
\bigcap_{i \in I} \operatorname{con} \phi_{x_{i}}^{\prime}(z) \quad \text { if } z \in U, \\
\left\{y_{0}\right\} \quad \text { if } z \notin U .
\end{array}\right.
$$

Then for each $z \in X, z \notin \operatorname{con} \Phi(z)$ by the following reason: in case $z \in U$, then $z \in N_{x_{i}}$ for some $i \in I$. Therefore $z \notin \operatorname{con} \phi_{x_{i}}^{\prime}(z)=\operatorname{con} \phi_{x_{i}}(z)$ so that $z \notin \operatorname{con} \Phi(z)$. On the other hand, in case $z \in X \backslash U$, then $z \in X \backslash D$. Therefore we have $z \neq y_{0}$, so that $z \notin \operatorname{con} \Phi(z)$.

Now we show that for each $y \in X, \Phi^{-1}(y)$ is open in $X$. Indeed, in case $y \neq y_{0}$, we have

$$
\begin{aligned}
\Phi^{-1}(y) & =\{z \in X: y \in \Phi(z)\} \\
& =\{z \in U: y \in \Phi(z)\} \cup\{z \in X \backslash U: y \in \Phi(z)\} \\
& =\{z \in U: y \in \Phi(z)\}=\left\{z \in U: y \in \bigcap_{i \in I} \operatorname{con} \phi_{x_{i}}^{\prime}(z)\right\} \\
& =U \cap\left[\bigcap_{i \in I}\left(\operatorname{con} \phi_{x_{i}}^{\prime}\right)^{-1}(y)\right]
\end{aligned}
$$

is open in $X$ since $\left(\operatorname{con} \phi_{x_{i}}^{\prime}\right)^{-1}(y)$ is open in $X$ for each $i \in I$.

In case $y=y_{0}$, we have

$$
\begin{aligned}
\Phi^{-1}\left(y_{0}\right) & =\left\{z \in X: y_{0} \in \Phi(z)\right\} \\
& =\left\{z \in U: y_{0} \in \Phi(z)\right\} \cup\left\{z \in X \backslash U: y_{0} \in \Phi(z)\right\} \\
& =\left[U \cap\left(\bigcap_{i \in I}\left(\operatorname{con} \phi_{x_{i}}^{\prime}\right)^{-1}\left(y_{0}\right)\right)\right] \cup(X \backslash U) \\
& =\left[U \cap\left(\bigcap_{i \in I}\left(\operatorname{con} \phi_{x_{i}}\right)^{-1}\left(y_{0}\right)\right)\right] \cup(X \backslash U) \\
& =\left[\bigcap_{i \in I}\left(\operatorname{con} \phi_{x_{i}}\right)^{-1}\left(y_{0}\right)\right] \cup(X \backslash U) .
\end{aligned}
$$

By the assumption and condition (i), for each $z \in X \backslash D$,

$$
y_{0} \in \operatorname{con} \phi(z) \subset \operatorname{con} \phi_{x_{i}}(z) \text { for each } i \in I \text {. }
$$

Therefore for each $i \in I, X \backslash D \subset\left(\operatorname{con} \phi_{x_{i}}\right)^{-1}\left(y_{0}\right)$, so that $X \backslash U \subset$ $\left(\operatorname{con} \phi_{x_{i}}\right)^{-1}\left(y_{0}\right)$. Therefore,

$$
\begin{aligned}
\Phi^{-1}\left(y_{0}\right) & =\left[\bigcap_{i \in I}\left(\operatorname{con} \phi_{x_{i}}\right)^{-1}\left(y_{0}\right)\right] \cup(X \backslash U) \\
& =\bigcap_{i \in I}\left(\operatorname{con} \phi_{x_{i}}\right)^{-1}\left(y_{0}\right)
\end{aligned}
$$

is open in $X$ since $\left(\operatorname{con} \phi_{x_{i}}\right)^{-1}\left(y_{0}\right)$ is open in $X$ for each $i \in I$. Therefore $\Phi: X \rightarrow 2^{X}$ is a correspondence of class $L$. 
Finally we shall show that for each $z \in X \backslash D, y_{0} \in \operatorname{con} \Phi(z)$. First, if $z \in X \backslash U$, then $y_{0} \in \operatorname{con} \Phi(z)=\left\{y_{0}\right\}$. For $z \in U \backslash D$, we must show that $y_{0} \in \operatorname{con} \Phi(z)$. Indeed, for every $i \in I$,

$$
\phi(z) \subset \phi_{x_{i}}^{\prime}(z)=\phi_{x_{i}}(z) \text { for each } z \in U,
$$

so that $\phi(z) \subset \Phi(z)$ for each $z \in U$. By the assumption, $y_{0} \in \operatorname{con} \phi(z) \subset$ $\operatorname{con} \Phi(z)$ for each $z \in U \backslash D$. Therefore, for all $z \in X \backslash D, y_{0} \in \operatorname{con} \Phi(z)$. Therefore by letting $X_{0}=\left\{y_{0}\right\}$, all the hypotheses of Lemma are satisfied, so that there exists a point $\tilde{x} \in X$ such that $\Phi(\tilde{x})=\varnothing$. This implies that $\tilde{x} \in U$. Since $\phi(z) \subset \Phi(z)$ for each $z \in U, \phi(\tilde{x})=\varnothing$, which is a contradiction. Hence there must exist a maximal element $\hat{x} \in X$ such that $\phi(\hat{x})=\varnothing$.

Q.E.D.

Remark. As we have seen in the previous example, the correspondence $\phi: X \rightarrow$ $2^{X}$ defined by

$$
\phi(x)=\left\{\begin{array}{l}
\left\{y \in X: 0 \leq y \leq x^{2}\right\}, \quad x \in(0,1), \\
\varnothing, \quad x=0,
\end{array}\right.
$$

is $L_{s}$-majorized but not of class $L$. Therefore any maximal element existence theorem for class $L$ (i.e., Lemma of Theorem 1 or results in $[13,23,24,26]$ ) cannot be applicable. However, by letting $D=\left\{y_{0}\right\}=\{0\}$ in Theorem 1, we have a maximal element $0 \in[0,1)$ such that $\phi(0)=\varnothing$.

In case $X$ is compact convex, we have the following immediate consequence which generalizes Fan's maximal element existence theorem appeared in [4]:

Corollary. Let $X$ be a nonempty compact convex subset of a Hausdorff topological vector space and let $\phi: X \rightarrow 2^{X}$ be a correspondence of class $L$; i.e., for each $x \in X, x \notin \operatorname{con} \phi(x)$ and for each $y \in X, \phi^{-1}(y)$ is open in $X$. Then there exists a point $\hat{x} \in X$ such that $\phi(\hat{x})=\varnothing$.

\section{EXISTENCE OF EQUILIBRIUM FOR A NONPARACOMPACT N-PERSON GAME}

As we have seen in $[4,5,6,18,23,24,26]$, maximal element existence theorems are essential tools in the proofs of the existence of equilibria for generalized games.

As an application of Theorem 1, we obtain the following existence theorem of equilibrium for a nonparacompact 1-person game with an $L_{s}$-majorized preference correspondence.

Theorem 2. Let $X$ be a nonempty convex subset of a Hausdorff topological vector space (a choice set), $D$ be a nonempty compact subset of $X$, and $y_{0} \in D$. Let $A, B: X \rightarrow 2^{X}$ be constraint correspondences and $P: X \rightarrow 2^{X}$ be a preference correspondence satisfying

(1) $A \cap P$ is $L_{s}$-majorized;

(2) for each $x \in X, A(x)$ is nonempty and $\operatorname{con} A(x) \subset B(x)$;

(3) for each $y \in X, A^{-1}(y)$ is open in $X$;

(4) the correspondence $\mathrm{cl} B: X \rightarrow 2^{X}$ is upper semicontinuous;

(5) for each $x \in X \backslash D, y_{0} \in(A \cap P)(x)$.

Then there exists an equilibrium choice $\hat{x} \in X$, i.e., $\hat{x} \in \operatorname{cl} B(\hat{x})$ and $A(\hat{x}) \cap$ $P(\hat{x})=\varnothing$. 
Proof. Let $F=\{x \in X: x \in \operatorname{cl} B(x)\}$; then $F$ is closed in $X$ since $\operatorname{cl} B$ is upper semicontinuous. Define $\psi: X \rightarrow 2^{X}$ by

$$
\psi(x)=\left\{\begin{array}{l}
\operatorname{con}(A \cap P)(x) \text { if } x \in F, \\
\operatorname{con} A(x) \text { if } x \notin F .
\end{array}\right.
$$

If $x \notin F$, then $X \backslash F$ is an open neighborhood of $x$ in $X$ such that for each $z \in X \backslash F, z \notin \operatorname{cl} B(z)$ and $\psi(z)=\operatorname{con} A(z)$; since con $A(z) \subset B(z)$, we have $z \notin \operatorname{con} A(z)$. And it is clear that $\psi(z) \subset \operatorname{con} A(z)$. Since $A^{-1}(y)$ is open in $X,(\operatorname{con} A)^{-1}(y)$ is also open in $X$ for all $y \in X$ by Lemma 5.1 in [26], so that $\operatorname{con} A$ is an $L_{s}$-majorant of $\psi$ at $x$.

Now suppose that $x \in F$ and $\psi(x)=\operatorname{con}(A \cap P)(x) \neq \varnothing$; then by the assumption (1), there exist $\phi_{x}: X \rightarrow 2^{X}$ and an open neighborhood $N_{x}$ of $x$ in $X$ such that

(i) $(A \cap P)(z) \subset \phi_{x}(z)$ for all $z \in X$,

(ii) $z \notin \operatorname{con} \phi_{x}(z) \quad$ for all $z \in N_{x}$,

(iii) $\phi_{x}^{-1}(y)$ is open in $X$ for all $y \in X$.

Define $\phi_{x}^{\prime}: X \rightarrow 2^{X}$ by

$$
\phi_{x}^{\prime}(z)= \begin{cases}\operatorname{con} \phi_{x}(z) \cap \operatorname{con} A(z), & \text { if } z \in F, \\ \operatorname{con} A(z), & \text { if } z \notin F .\end{cases}
$$

Then we have the following facts: (a) for each $z \in N_{x}$, it is easy to see that $\psi(z) \subset \phi_{x}^{\prime}(z)$, (b) for each $z \in N_{x}, z \notin \operatorname{con} \phi_{x}^{\prime}(z)$, and (c) for each $y \in X$,

$$
\begin{aligned}
\left(\phi_{x}^{\prime}\right)^{-1}(y) & =\left\{z \in X: y \in \phi_{x}^{\prime}(z)\right\} \\
& =\left\{z \in F: y \in \phi_{x}^{\prime}(z)\right\} \cup\left\{z \in X \backslash F: y \in \phi_{x}^{\prime}(z)\right\} \\
& =\left\{z \in F: y \in\left[\operatorname{con} \phi_{x}(z) \cap \operatorname{con} A(z)\right]\right\} \cup\{z \in X \backslash F: y \in \operatorname{con} A(z)\} \\
& =\left[F \cap(\operatorname{con} A)^{-1}(y) \cap\left(\operatorname{con} \phi_{x}\right)^{-1}(y)\right] \cup\left[(X \backslash F) \cap(\operatorname{con} A)^{-1}(y)\right] \\
& =\left[\left(\operatorname{con} \phi_{x}\right)^{-1}(y) \cup(X \backslash F)\right] \cap(\operatorname{con} A)^{-1}(y)
\end{aligned}
$$

is open in $X$.

This shows that $\phi_{x}^{\prime}$ is an $L_{s}$-majorant of $\psi$ at $x$. Therefore $\psi$ is $L_{s}$ majorized. By Theorem 1, there exists a point $\hat{x} \in X$ such that $\psi(\hat{x})=\varnothing$. Since $A(x) \neq \varnothing$ for each $x \in X$, we must have $\hat{x} \in F$, i.e., $\hat{x} \in \operatorname{cl} B(\hat{x})$ and $\psi(\hat{x})=\operatorname{con}(A \cap P)(\hat{x})=\varnothing$, so that $A(\hat{x}) \cap P(\hat{x})=\varnothing$. Q.E.D.

In case $X$ is compact convex and $A=B$, by letting $D=X$ we have the following immediate consequence of Theorem 2:

Corollary 1. Let $X$ be a nonempty compact convex subset of a Hausdorff topological vector space and let $P, A: X \rightarrow 2^{X}$ be two correspondences satisfying the following conditions:

(1) $A \cap P$ is $L_{s}$-majorized;

(2) for each $x \in X, A(x)$ is nonempty convex;

(3) for each $y \in X, A^{-1}(y)$ is open in $X$;

(4) the correspondence $\operatorname{cl} A: X \rightarrow 2^{X}$ is upper semicontinuous.

Then there exists an equilibrium choice $\hat{x} \in X$, i.e., $\hat{x} \in \operatorname{cl} A(\hat{x})$ and $A(\hat{x}) \cap$ $P(\hat{x})=\varnothing$.

Let $I$ be a finite set of agents and $X_{i}$ be a Hausdorff topological vector space. Let $X=\prod_{i \in I} X_{i}$. For a given correspondence $A_{i}: X \rightarrow 2^{X_{i}}$, recall 
that a correspondence $A_{i}^{\prime}: X \rightarrow 2^{X}$ is defined by $A_{i}^{\prime}(x)=\left\{y \in X: y_{i} \in A_{i}(x)\right\}$ (= $\pi_{i}^{-1}\left(A_{i}(x)\right)$ where $\pi_{i}: X \rightarrow X_{i}$ is the $i$ th projection). Then it is easy to show that the following two conditions are equivalent:

(1) $A_{i}^{\prime}$ is a correspondence of class $L$;

(2) for each $x \in X, x_{i} \notin \operatorname{con} A_{i}(x)$ and for each $y \in X_{i}, A_{i}^{-1}(y)$ is open in $X$.

We shall now show that the case of $\mathrm{n}$ agents $(n>1)$ in a nonparacompact abstract economy with preference correspondences of class $L$ can be reduced to a 1-person game with $L_{s}$-majorized preference correspondence (i.e., Theorem 2).

Theorem 3. Let $\Gamma=\left(X_{i}, A_{i}, B_{i}, P_{i}\right)_{i \in I}$ be an abstract economy where $I$ is a finite set such that for each $i \in I$,

(1) $X_{i}$ is a nonempty convex subset of a Hausdorff topological vector space and $D_{i}$ is a nonempty compact subset of $X_{i}$

(2) for each $x \in X=\prod_{i \in I} X_{i}, A_{i}(x)$ is nonempty and con $A_{i}(x) \subset B_{i}(x)$;

(3) for each $y \in X_{i}, A_{i}^{-1}(y)$ is open in $X$;

(4) the correspondence $\mathrm{cl} B_{i}: X \rightarrow 2^{X_{i}}$ is upper semicontinuous;

(5) the correspondence $A_{i}^{\prime} \cap P_{i}^{\prime}$ is of class $L$;

(6) there exists a point $y_{0} \in \prod_{i \in I} D_{i}$ such that for each $x \in X \backslash \prod_{i \in I} D_{i}$, $y_{0} \in\left(A_{i}^{\prime} \cap P_{i}^{\prime}\right)(x)$.

Then $\Gamma$ has an equilibrium choice $\hat{x} \in X$, i.e., for each $i \in I, \hat{x}_{i} \in \operatorname{cl} B_{i}(\hat{x})$ and $A_{i}(\hat{x}) \cap P_{i}(\hat{x})=\varnothing$.

Proof. Let $D=\prod_{i \in I} D_{i}$. Then $D$ is compact by Tychonoff's product theorem. Define the correspondences $A, B, P: X \rightarrow 2^{X}$ by

$$
A(x)=\prod_{i \in I} A_{i}(x), \quad B(x)=\prod_{i \in I} B_{i}(x),
$$

and

$$
P(x)= \begin{cases}\bigcap_{i \in I(x)} P_{i}^{\prime}(x), & \text { if } I(x) \neq \varnothing, \\ \varnothing, & \text { if } I(x)=\varnothing,\end{cases}
$$

where $I(x)=\left\{i \in I: A_{i}(x) \cap P_{i}(x) \neq \varnothing\right\}$.

By (2), for each $x \in X, A(x)$ is nonempty and con $A(x) \subset B(x)$. By (3), for each $y \in X, A^{-1}(y)=\bigcap_{i \in I} A_{i}^{-1}(y)$ is open in $X$. Moreover, since $\operatorname{cl} B(x)=$ $\operatorname{cl} \prod_{i \in I} B_{i}(x)=\prod_{i \in I} \operatorname{cl} B_{i}(x)$ for each $x \in X$, by (4) and Lemma 3 in [7], $\operatorname{cl} B: X \rightarrow 2^{X}$ is also upper semicontinuous. By (6), for each $x \in X \backslash D, I(x)=$ $I$ and $y_{0} \in(A \cap P)(x)$. Therefore the hypotheses (2), (3), (4), and (5) of Theorem 2 are satisfied. Finally we shall show that the correspondence $A \cap P$ is $L_{s}$-majorized. Let $x \in X$ with $(A \cap P)(x) \neq \varnothing$, i.e., $\prod_{i \in I(x)}\left(A_{i} \cap P_{i}\right)(x) \neq \varnothing$ and $I(x) \neq \varnothing$. We first show that there exists an open neighborhood $N_{x}$ of $x$ in $X$ such that $I(x) \subset I(z)$ (and hence $I(z) \neq \varnothing$ ) for all $z \in N_{x}$. Indeed, let $i \in I(x)$; as $A_{i}^{\prime}(x) \cap P_{i}^{\prime}(x) \neq \varnothing$, take any $y \in A_{i}^{\prime}(x) \cap P_{i}^{\prime}(x)$, then $x \in$ $\left(A_{i}^{\prime} \cap P_{i}^{\prime}\right)^{-1}(y)$. Let $N_{i}=\left(A_{i}^{\prime} \cap P_{i}^{\prime}\right)^{-1}(y)$, then by assumption (5), $N_{i}$ is an open neighborhood of $x$. Let $N_{x}=\bigcap_{i \in I(x)} N_{i}$, then $N_{x}$ is an open neighborhood of $x$ in $X$. If $z \in N_{x}$, then for each $i \in I(x), z \in N_{i}=\left(A_{i}^{\prime} \cap P_{i}^{\prime}\right)^{-1}(y)$ so that 
$y \in\left(A_{i}^{\prime} \cap P_{i}^{\prime}\right)(z)$ and hence $\left(A_{i}^{\prime} \cap P_{i}^{\prime}\right)(z) \neq \varnothing$; that is, $i \in I(z)$. This shows that $I(x) \subset I(z)$ for all $z \in N_{x}$. Now fix any $i_{0} \in I(x)$. Then for any $z \in N_{x}$, we have

$$
\begin{aligned}
(A \cap P)(z) & \subset \bigcap_{i \in I(z)} A_{i}^{\prime}(z) \cap P_{i}^{\prime}(z) \\
& \subset \bigcap_{i \in I(x)} A^{\prime}(z) \cap P^{\prime}(z) \quad(\text { since } I(x) \subset I(z)) \\
& \subset\left(A_{i_{0}}^{\prime} \cap P_{i_{0}}^{\prime}\right)(z) .
\end{aligned}
$$

Since $X$ is regular and $N_{x}$ is an open neighborhood of $x$ in $X$, there exists an open neighborhood $M_{x}$ of $x$ in $X$ such that $M_{x} \subset \operatorname{cl} M_{x} \subset N_{x}$.

We now define a correspondence $\phi_{x}: X \rightarrow 2^{X}$ by

$$
\phi_{x}(z)= \begin{cases}\left(A_{i_{0}}^{\prime} \cap P_{i_{0}}^{\prime}\right)(z), & \text { if } z \in \operatorname{cl} M_{x}, \\ X, & \text { if } z \notin \operatorname{cl} M_{x} .\end{cases}
$$

Then it is easy to show that $(A \cap P)(z) \subset \phi_{x}(z)$ for each $z \in X$. And, for each $z \in M_{x}, z \notin \operatorname{con} \phi_{x}(z)$ by assumption (5). For each $y \in X$,

$$
\begin{aligned}
\phi_{x}^{-1}(y) & =\left\{z \in X: y \in \phi_{x}(z)\right\} \\
& =\left\{z \in \operatorname{cl} M_{x}: y \in \phi_{x}(z)\right\} \cup\left\{z \in X \backslash \operatorname{cl} M_{x}: y \in \phi_{x}(z)\right\} \\
& =\left\{z \in \operatorname{cl} M_{x}: y \in\left(A_{i_{0}}^{\prime} \cap P_{i_{0}}^{\prime}\right)(z)\right\} \cup\left(X \backslash \operatorname{cl} M_{x}\right) \\
& =\left[\operatorname{cl} M_{x} \cap\left(A_{i_{0}}^{\prime} \cap P_{i_{0}}^{\prime}\right)^{-1}(y)\right] \cup\left(X \backslash \operatorname{cl} M_{x}\right) \\
& =\left(A_{i_{0}}^{\prime} \cap P_{i_{0}}^{\prime}\right)^{-1}(y) \cup\left(X \backslash \operatorname{cl} M_{x}\right)
\end{aligned}
$$

is open in $X$ by (5). Therefore $\phi_{x}$ is an $L_{s}$-majorant of $A \cap P$ at $x$. (Note that $M_{x}$ is a desired open neighborhood of $x$ in $X$.)

Therefore $A \cap P$ is $L_{s}$-majorized, so that all the hypotheses of Theorem 2 are satisfied. By Theorem 2, there exists an equilibrium point $\hat{x} \in X$ such that $\hat{x}_{i} \in \operatorname{cl} B(\hat{x})$ and $A(\hat{x}) \cap P(\hat{x})=\varnothing$. Hence for each $i \in I, \hat{x}_{i} \in \operatorname{cl} B_{i}(\hat{x})$. Next we shall show that $I(\hat{x})=\varnothing$. Suppose $I(\hat{x}) \neq \varnothing$. Since $A(\hat{x}) \cap P(\hat{x})=\varnothing$ implies $A_{i}(\hat{x}) \cap P_{i}(\hat{x})=\varnothing$ for at least one $i \in I(\hat{x})$, which contradicts the definition of $I(\hat{x})$. Therefore we must have $I(\hat{x})=\varnothing$, i.e., $A_{i}(\hat{x}) \cap P_{i}(\hat{x})=\varnothing$ for each $i \in I$. Q.E.D.

Remarks. (1) Since $X_{i}$ is neither compact nor paracompact, our Theorem 3 is comparable with the privious equilibrium existence results in $[4,5,6,18,23$, $24,26]$ and as remarked before, equilibrium existence theorems for compact (or paracompact) abstract economies cannot be applied to our settings.

(2) We do not know whether assumption (5) can be relaxed to $L_{s}$-majorized correspondences and we also do not know whether the set of agents $I$ may be infinite.

In case $X_{i}$ is compact convex and $A_{i}=B_{i}$ for each $i \in I$, by letting $D_{i}=X_{i}$ for each $i \in I$, we have the following immediate consequence of Theorem 3 :

Corollary 2. Let $\Gamma=\left(X_{i}, A_{i}, P_{i}\right)_{i \in I}$ be an abstract economy where $I$ is a finite set such that for each $i \in I$,

(1) $X_{i}$ is a nonempty compact convex subset of a Hausdorff topological vector space;

(2) for each $x \in X=\prod_{i \in I} X_{i}, A_{i}(x)$ is a nonempty convex subset of $X_{i}$; 
(3) for each $y \in X_{i}, A_{i}^{-1}(y)$ is open in $X$;

(4) the correspondence $\mathrm{cl} A_{i}: X \rightarrow 2^{X_{i}}$ is upper semicontinuous;

(5) the correspondence $A_{i}^{\prime} \cap P_{i}^{\prime}$ is of class $L$.

Then $\Gamma$ has an equilibrium choice $\hat{x} \in X$, i.e., for each $i \in I, \hat{x}_{i} \in \operatorname{cl} A_{i}(\hat{x})$ and $A_{i}(\hat{x}) \cap P_{i}(\hat{x})=\varnothing$.

When the set of agents $I$ is finite, Corollary 2 answers the question raised in [26, p. 243] positively; i.e., Corollary 2 holds true in nonmetrizable and nonlocally convex spaces.

\section{REFERENCES}

1. C. Aliprantis and D. Brown, Equilibria in markets with a Riesz space of commodities, J. Math. Econom. 11 (1983), 189-207.

2. K. J. Arrow and G. Debreu, Existence of an equilibrium for a competitive economy, Econometrica 22 (1954), 265-290.

3. T. F. Bewley, Existence of equilibria in economies with infinitely many commodities, J. Econom. Theory 4 (1972), 514-540.

4. A. Borglin and $\mathrm{H}$. Keiding, Existence of equilibrium actions and of equilibrium: $A$ note on the 'new' existence theorem, J. Math. Econom. 3 (1976), 313-316.

5. X. P. Ding, W. K. Kim, and K.-K.Tan, Equilibria of non-compact generalized games with $L^{*}$ majorized preferences, J. Math. Anal. Appl., in press.

6. __ Eqilibrium theorems on non-compact abstract economies with $L^{*}$-majorized preference correspondences, in preparation.

7. K. Fan, Fixed-point and minimax theorems in locally convex topological linear spaces, Proc. Nat. Acad. Sci. U.S.A. 38 (1952), 131-136.

8. _ _ A generalization of Tychonoff's fixed point theorem, Math. Ann. 142 (1961), 305-310.

9. 519-537.

10. P. Fishburn and R. Rosenthal, Noncooperative games and nontransitive preferences, Math. Social Sci. 12 (1986), 1-7.

11. M. Florenzano, On the existence of equilibria in economies with an infinite dimensional commodity spaces, J. Math. Econom. 12 (1983), 207-219.

12. _ On an extension of the Gale-Nikaido-Debreu lemma, Econom. Lett. 25 (1987), 51-53.

13. D. Gale and A. Mas-Colell, An equilibrium existence for a general model without ordered preferences, J. Math. Econom. 2 (1975), 9-15.

14. A. Kajii, Note on equilibrium without ordered preferences in topological vector space, Econom. Lett. 27 (1988), 1-4.

15. H. Keiding, Existence of economic equilibriums: New results and open problems, Lecture Notes in Econom. and Math. System, vol. 226, Springer-Verlag, Berlin and New York, 1984, pp. 223-243.

16. M. Ali Khan, On extensions of the Cournot-Nash theorem, Advances in Equilibrium Theory (C. Aliprantis et al., eds.), Springer-Verlag, Berlin, 1985.

17. A. Mas-Colell, An equilibrium existence theorem without complete or transitive preferences, J. Math. Econom. 1 (1974), 237-246.

18. G. Mehta, Fixed points, equilibria and maximal elements in linear topological spaces, Comm. Math. Univ. Carolinae 28 (1987), 377-385.

19. W. Shafer and H. Sonnenschein, Equilibrium in abstract economies without ordered preferences, J. Math. Econom. 2 (1975), 345-348.

20. W. Shafer, Equilibrium in economies without ordered preferences or free disposal, J. Math. Econom. 3 (1976), 135-137.

21. H. Sonnenschein, Demand theory without transitive preference with applications to the theory of competitive equilibrium, Preferences, Utility and Demand (J. Chipman et al., eds.), Harcourt Brace Jovanovich, New York, 1971. 
22. E. Tarafdar and G. Mehta, A generalized version of the Gale-Nikaido-Debreu theorem, Comm. Math. Univ. Carolinae 28 (1987), 655-659.

23. S. Toussaint, On the existence of equilibria in economies with infinitely many commodities and without ordered preferences, J. Econom. Theory 33 (1984), 98-115.

24. C. I. Tulcea, On the equilibriums of generalized games, The Center for Mathematical Studies in Economics and Management Science, Paper No. 696, 1986.

25. _ On the approximation of upper semicontinuous correspondences and the equilibrium of the generalized games, J. Math. Anal. Appl. 136 (1988), 267-289.

26. N. C. Yannelis and N. D. Prabhakar, Existence of maximal elements and equilibria in linear topological spaces, J. Math. Econom. 12 (1983), 233-245.

27. _ Erratum, J. Math. Econom. 13 (1984), 305.

28. N. C. Yannelis, Maximal elements over non-compact subsets of linear topological spaces, Econom. Lett. 17 (1985), 133-136.

29. __ On a market equilibrium theorem with an infinite number of commodities, J. Math. Anal. Appl. 108 (1985), 595-599.

Department of Mathematics Education, Chungbuk National University, Cheongue 360-763, KOREA

E-mail address: wkkim@cbucc.cbnu.ac.kr 